\title{
Space-Time Super-Modulation and its Application to Joint Medium Access and Rateless Transmission
}

\author{
Konstantinos Nikitopoulos, Farhad Mehran \\ Institute for Communication Systems (ICS) \\ Home of 5G Innovation Center \\ University of Surrey, Guildford, UK \\ Email: \{k.nikitopoulos,f.mehran\}@ surrey.ac.uk
}

\author{
Hamid Jafarkhani \\ Henry Samueli School of Engineering \\ Center for Pervasive Communications and Computing \\ University of California, Irvine, CA \\ Email: hamidj@uci.edu
}

\begin{abstract}
The paper introduces the concept of Space-Time Super-Modulation according to which additional low rate and highly reliable information can be transmitted by further supermodulating blocks of traditionally modulated and space-time encoded information. This is achieved by exploiting the redundant information introduced by the space-time-block codes and, specifically, by efficiently mapping transmission patterns to specific information content. It is shown that Space-Time SuperModulation can be efficiently used in the context of MachineType-Communications to enable joint medium access and rateless data transmission while minimizing or even eliminating the need for transmitting preamble sequences. Compared with traditional approaches that use encoded preambles or preambles based on Zadoff-Chu sequences to transmit the signature information of transmitted packets, Space-Time Super-Modulation can achieve throughput gains of more than $45 \%$ when transmitting blocks of 200 symbols.
\end{abstract}

Index Terms-Multiple-input multiple-output (MIMO), Spacetime block coding (STBC), Machine Type Communications (MTCs), Multilevel codes.

\section{INTRODUCTION}

As telecommunication technologies and applications evolve, a continuously increasing number of devices require to be connected wirelessly. Such Machine Type Communications (MTCs) have diverse requirements depending on the service, the application, and the type of devices that need to communicate [1]. These diverse requirements, together with the expected number of devices to be connected during the coming years, introduce new challenges and trigger a need to revisit the current medium access and data transmission strategies [2][3][4].

One of the main MTC challenges relates to the sporadic wireless traffic which is expected to dramatically increase in the near future [4][5]. In sporadic data transmission, a small amount of information is typically transmitted. Then, the signaling overhead required to connect (and synchronize) a machine, together with the signaling required for reliable transmission, can result in severe network underutilization. For example, for Random Access Channel (RACH) as used in LTE/LTE-A, to transmit 100 bytes of data from a user to the Base Station (BS), the signaling procedure requires approximately 159 and 136 bytes of overhead in the uplink and downlink respectively [1]. To avoid this overhead, as well as the delays induced from such an information exchange, recent research focuses on finding solutions able to simultaneously handle medium access and data transmission (i.e., users payload) [6] that are referred to as "one-shot transmission" [7] or "joint medium access and data transmission techniques".

Ideally, a future MTC protocol should enable one-shot, asynchronous, and highly-reliable transmission, with very low (or no) signaling overhead. However, reliability and low signaling overhead are, in principle, competing requirements. For example, for recovering the transmitted information of a specific user it is necessary for the receiver to reliably identify its identification (ID) information. This can prove to be very challenging, especially in unfavorable transmission environments of high dynamics (e.g., car communications), and/or very low signal-to-noise ratio (SNR) operational environment (e.g., in cases where high coverage is targeted) for which the ID information should be protected with very strong codes, or long preamble transmissions, that involves heavier ID signaling.

In addition to ID transmission, in order to efficiently transmit information close to the capabilities of the transmission channel (i.e., close to channel capacity) efficient rate adaptation is required [8] that takes place at the transmitter side. Current rate adaption schemes that are based on adaptive modulation and coding require instantaneous knowledge of the channel condition and add undesirable signaling overhead [4]. This overhead can become significantly higher if information is transmitted over different coherence times. Rateless codes applied to the physical (PHY) layer are a very promising way to alleviate the need for this overhead. However, for decoding of rateless codes the receiver needs to know not only the ID of the machine, but also the ordered position of the received packet in the whole rateless-coded packet, which would typically require additional signaling.

In order to avoid long packet ID transmission, the idea of jointly coding the machine header and payload has been highlighted for future wireless networks [1]. However, to the best of the authors' knowledge, no practical solutions have been proposed so far. This work introduces a SpaceTime Super-Modulation (STSM) scheme that enables highlyreliable joint medium access and rateless transmission, without requiring the transmission of preambles for delivering the signature information of a transmitted packet. STSM assumes 
multiple transmit and receive antenna (MIMO) systems where space-time-block coding [9][10][11] is employed to increase transmission reliability and exploits the redundancy introduced from the space-time-block code to jointly encode the signature information of the transmitted packet together with the useful data information. STSM performs encoding of the signature information by altering the pattern of the transmitted spacetime-encoded packet, in a way that the Euclidean distance is increased between possible signature words. The data information is encoded by means of "traditional" (e.g., rateless) binary codes. STSM can be assumed to be a member of the greater family of multilevel codes [12]. However, in contrast to Trellis-Coded-Modulation (TCM) [13], STSM allows transmitting the signature information by using flexible transmission rates and, therefore, it enables flexible detection reliability. In addition, contrary to Imai's originally proposed idea of multilevel coding [14], the signature bits and the data bits are not separately encoded by using binary codes that are cooptimized for maximizing the minimum Euclidean distance of the codewords. Instead, STSM uses traditional binary channel coding and space-time coding for the data, while, it encodes the low rate signature information by exploiting the structure of the space-time encoded block. This allows using STSM with any kind of data coding. To the best of the authors' knowledge, STSM is the first multilevel coding approach, exploiting spacetime-block codes. In addition, it is the first time that such an approach is used to enable of "rateless" coding in MTC communications.

The rest of the paper is organized as follows. In Section II the concept of STSM is presented. Section III discusses how STSM can be used for joint data medium access and data transmission, and in Section IV the evaluation of the proposed approach follows.

\section{Space-Time Super-Modulation (STSM)}

Typically, the transmission pattern of the conventionally modulated symbols after space-time block coding, i.e., the phase, the amplitude and the relative position of the actual and redundant information in the space/time/phase grid) is unique, predetermined, and a-priori known to both the transmitter and the receiver [9][10]. Instead of having such a unique pattern, STSM allows the employment of multiple but still predefined sets of Super Modulation Patterns (SMPs). Which pattern will be transmitted is finally dictated by the additional information to be transmitted after appropriate bit-to-pattern (similar to the traditional bit-to-symbol) mapping. If the transmitted pattern can be reliably identified at the receiver side, the corresponding information content can be recovered and therefore a throughput increase can be achieved. In other words, the additional bits are encoded using the corresponding SMPs in such a way that the corresponding minimum Euclidean distance between those patterns is maximized.

Various approaches can be used to super-modulate the conventionally modulated symbols as a function of the corresponding SMP as long as the corresponding pattern can be uniquely identified (i.e, demodulated) at the receiver side.
For example, the SMP can modulate the phase and/or the amplitude of conventionally modulated symbols, the relative position of the actual and redundant information (in the case of space-time block codes), or even a combination one of those parameters. This paper focuses in the case of phase STSM due to its simplicity and since, in contrast to amplitude modulation methods, it avoids increasing the peak to average power ratio which makes the detection efficiency very sensitive to the nonlinear devices of the processing loop (e.g., digital to analog converter, high power amplifier).

In the rest of this section the encoding and decoding process of STSM are presented. While the proposed approach is applicable to any type of space-time block code, the practical $2 \times 2$ Alamouti space-time code [9] is examined, especially since for MTCs a low number of antennas is expected, and the discussion is focused on low rate order constant amplitude constellations (e.g., BPSK), since as previously discussed the Super Modulation (SM) scheme primarily targets "unfavorable" transmission scenarios.

\section{A. STSM Encoding}

The STSM scheme requires transmission in blocks. The size of the block is assumed to be equal to $L$ channel uses, such that the corresponding transmission channel can be assumed static for the block duration. The proposed STSM scheme is depicted in Fig. 1, for the case of a $2 \times 2$ Alamouti scheme. For each transmitted STSM block the bits to be transmitted are split into two subsets: (a) The Conventionally Modulated Bits (CMB) subset and (b) The Super-Modulated Bits (SMB) subset. The CMB subset consists of the bits which would typically be transmitted without STSM. These bits are mapped onto conventional complex information symbols $\mathbf{S}$. The SMB subchannel is of lower rate, and therefore of higher reliability than the $\mathrm{CMB}$, and consists of the additional bits to be transmitted via the proposed mapping technique. In MTCs this subchannel is used to transmit each packet's signature bits. The SMBs are mapped onto patterns (SMPs) via an appropriate SMB-to-SMP mapping. Then, the selected SMP $c$, which is characterized by its characteristic SMP vector $\boldsymbol{\Pi}_{c}$, determines the way that the conventionally produced symbols (from CMBs) will be further modulated via SM. After SM, the produced symbols are space-time encoded to produce $\mathbf{S}_{\mathbf{c}}$ which will be finally transmitted.

1) $C M B$ to Conventional Symbol Mapping: Since for a $2 \times 2$ scheme with Alamouti space-time code $B=L / 2$ channel uses deliver actual information and $B$ channel uses are related to the same information, $2 B \log _{2}|\mathcal{S}|$ bits can be mapped onto conventional complex information symbols $s_{i, b}$ drawn from a PSK or QAM constellation $\mathcal{S}$ of cardinality $|\mathcal{S}|$, with $i=$ 1,2 denoting the antenna index and $b=1, \ldots, B$. Then, the conventionally modulated word is

$$
\mathbf{S}=\left[\begin{array}{lll}
s_{1,1} & \ldots & s_{1, B} \\
s_{2,1} & \ldots & s_{2, B}
\end{array}\right]^{T} .
$$

2) SMB to Super-Modulation-Pattern (SMP) Mapping: If $C$ SMPs are available, $\log _{2}\left(\lfloor C\rfloor_{2^{N}}\right)$ SMBs are transmitted 


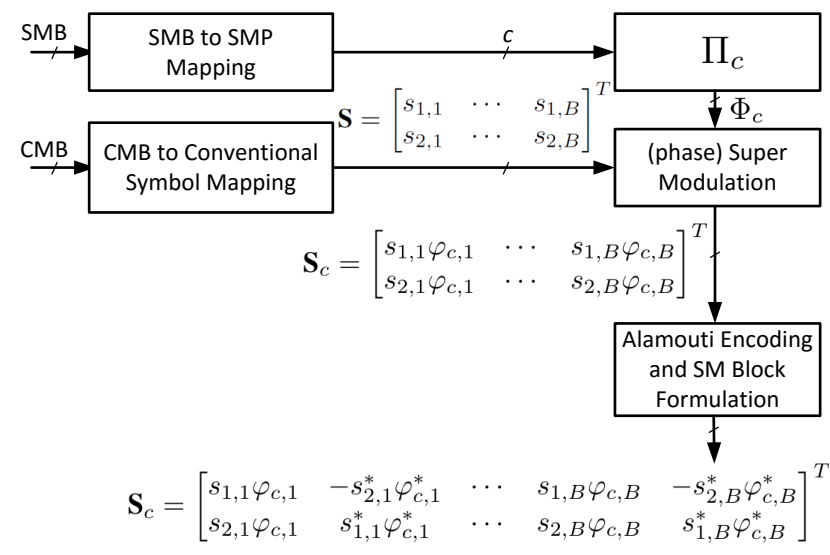

Fig. 1. Phase STSM scheme for $2 \times 2$ Alamouti space-time block code.

per block with an appropriate SMB-to-SMP mapping, with $\lfloor C\rfloor_{2^{N}}$ being the maximum power of 2 not exceeding $C$.

3) Phase Super-Modulation: Each SMP $c$ is related to a unique characteristic SMP vector $\Pi_{c}$ of length $B$. This vector is introduced to describe how the produced complex information symbols will be super-modulated. To produce the super-modulated symbols, it is assumed that each symbol can be further modulated by using one of the $M_{S M}$, predefined super-modulation states. For phase STSM these states are predefined distinct phase rotations. Then, if $c$ is the SMP to be transmitted, the symbols $s_{i, b}$, with $i=1,2$ and $b=1, \ldots, B$ will be super-modulated using the SM state (e.g. phase rotations) given by the $b$-th element of $\boldsymbol{\Pi}_{c}$. For example, if a phase super-modulation scheme with $M_{S M}=2$ modulation states (i.e., available phase rotations) is employed and if $\Pi_{c}(4)=2$, the symbols $s_{1,4}$ and $s_{2,4}$ will be phase super-modulated by using the 2nd available phase rotation.

More specifically, with phase super-modulation, the resulting symbol is

$$
s_{i, b}^{(c)}=s_{i, b} \varphi_{c, b} .
$$

For symmetric, $M$-PSK modulations, with the minimum phase distance between symbol constellations being $\phi_{\min }=2 \pi / M$, the phase rotation can be

$$
\varphi_{c, b}=\exp \left\{j \phi_{c, b}\right\}=\exp \left\{j \frac{2 \pi}{M_{S M} M}\left[\boldsymbol{\Pi}_{c}(b)-1\right]\right\} .
$$

It is noted that the phase rotations are such that the phase modulated symbols over different $b=1, \ldots, B$ do not coincide for any possible conventionally transmitted symbol. This attribute makes the different SMPs distinguishable at the receiver side.

\section{B. Alamouti Encoding and SM Block Formulation}

Eventually, according to the Alamouti space-time code, the corresponding redundant information for each pair of the $s_{i, b}^{(c)}$ symbols over the different $b$ indexes is calculated as an orthogonal transformation of these symbols. Then, without their exact positioning affecting the performance of the proposed scheme, we assume that the actual information of the $b$-th pair of symbols is transmitted over the $t=2 b-1$ channel use and the corresponding redundant information is transmitted over the $t=2 b$ channel use. Therefore, the transmitted supermodulated word employing the $c$-th pattern is

$$
\begin{gathered}
\mathbf{S}_{c}=\left[\begin{array}{ccccc}
s_{1,1}^{(c)} & -s_{2,1}^{(c) *} & \ldots & s_{1, B}^{(c)} & -s_{2, B}^{(c) *} \\
s_{2,1}^{(c)} & s_{1,1}^{(c) *} & \ldots & s_{2, B}^{(c)} & s_{1, B}^{(c) *}
\end{array}\right]^{T} \\
{\left[\begin{array}{ccccc}
s_{1,1} \varphi_{c, 1} & -s_{2,1}^{*} \varphi_{c, 1}^{*} & \ldots & s_{1, B} \varphi_{c, B} & -s_{2, B}^{*} \varphi_{c, B}^{*} \\
s_{2,1} \varphi_{c, 1} & s_{1,1}^{*} \varphi_{c, 1}^{*} & \ldots & s_{2, B} \varphi_{c, B} & s_{1, B}^{*} \varphi_{c, B}^{*}
\end{array}\right]^{T}}
\end{gathered}
$$

with $\varphi_{c, b}$ given by (3). Then, it can be easily verified that the proposed scheme preserves the Alamouti space-time code structure and therefore the corresponding diversity gain.

It is noted, that for this work, the selection of SMPs has been done in a way that the corresponding minimum Euclidean distance is (approximately) maximized via extensive simulations.

\section{STSM Receiver Processing}

As discussed, the transmission channel $\mathbf{H}$ consisting of the sub-channels $H_{m, n}$, from Tx antenna $m$ to Rx antenna $n$, is assumed static for the duration of a block transmission. The received $2 \times 2 B$ signal $\mathbf{Y}$ can be described as

$$
\mathbf{Y}=\mathbf{S}_{c} \mathbf{H}+\mathbf{N}
$$

where $\mathbf{N}$ is the $2 \times 2 B$ noise matrix consisting of independent and identically distributed (i.i.d.), zero-mean, complex Gaussian samples with variance $2 \sigma_{n}^{2}$. Then, the ML detector of the transmitted word is given by

$$
\hat{\mathbf{S}}_{c}=\arg \min _{\mathbf{S}_{c} \in \mathcal{W}}\left\{\mathcal{M}\left(\mathbf{S}_{c}\right)\right\}
$$

with

$$
\mathcal{M}\left(\mathbf{S}_{c}\right)=\left\|\mathbf{Y}-\mathbf{S}_{c} \mathbf{H}\right\|^{2} .
$$

and $\mathcal{W}$ being the set of all possible super-modulated words, The above minimization problem typically involves exhaustive calculation over all possible words, namely, over all possible transmitted symbols and SMPs, which is typically of prohibitive complexity. In order to reduce Rx complexity it can be easily shown after some algebraic manipulations that for a specific SMP $c$, the corresponding ML metric $\mathcal{M}\left(\mathbf{S}_{c}\right)$ can be expressed as

$$
\mathcal{M}\left(\mathbf{S}_{c}\right)=\sum_{b=1}^{B}\left\|\tilde{\mathbf{Y}}_{b}-\varphi_{c, b} \tilde{\mathbf{H}} \tilde{\mathbf{S}}_{b}\right\|^{2}
$$

with $\tilde{\mathbf{Y}}_{b}=\left[\begin{array}{llll}\mathbf{Y}[2 b-1,1] & \mathbf{Y}^{*}[2 b, 1] & \mathbf{Y}[2 b-1,2] & \mathbf{Y}^{*}[2 b, 2\end{array}\right]^{T}$, $\tilde{\mathbf{S}}_{b}=\left[\begin{array}{ll}s_{1, b} & s_{2, b}\end{array}\right]^{T}$ and

$$
\tilde{\mathbf{H}}=\left[\begin{array}{ll}
\tilde{\mathbf{h}}_{1} & \tilde{\mathbf{h}}_{2}
\end{array}\right]=\left[\begin{array}{cc}
H_{1,1} & H_{2,1} \\
H_{2,1}^{*} & -H_{1,1}^{*} \\
H_{1,2} & H_{2,2} \\
H_{2,2}^{*} & -H_{1,2}^{*}
\end{array}\right] .
$$

Then, since the terms summed in (7) are independent of each other, the corresponding minimization can be achieved through the minimization of each term. Then, the conventionally 
modulated symbols which minimize $\mathcal{M}\left(\mathbf{S}_{c}\right)$ for a give $c$ can be calculated as

$$
\begin{gathered}
\hat{\tilde{\mathbf{S}}}_{b}=\arg \min _{\tilde{\mathbf{S}}_{b} \in \mathcal{S}^{2}} \mathcal{M}_{c, b}= \\
\arg \min _{\tilde{\mathbf{S}}_{b} \in \mathcal{S}^{2}}\left\|\tilde{\mathbf{Y}}_{b}-\varphi_{c, b} \tilde{\mathbf{H}} \tilde{\mathbf{S}}_{b}\right\|^{2}, \forall b=1, \ldots, B .
\end{gathered}
$$

The corresponding minimum metric value for the specific SMP $c$ is hence calculated as

$$
\begin{gathered}
\mathcal{M}_{\text {min }}(c)=\min \left\{\sum_{b=1}^{B}\left\|\tilde{\mathbf{Y}}_{b}-\varphi_{c, b} \tilde{\mathbf{H}} \tilde{\mathbf{S}}_{b}\right\|^{2}\right\}= \\
\sum_{b=1}^{B}\left\|\tilde{\mathbf{Y}}_{b}-\varphi_{c, b} \tilde{\mathbf{H}} \tilde{\tilde{\mathbf{S}}}_{b}\right\|^{2} .
\end{gathered}
$$

The exhaustive search over all possible constellation symbols in (8) can be avoided by QR decomposition of the channel $\tilde{\mathbf{H}}$ as

$$
\tilde{\mathbf{H}}=\mathbf{Q}\left[\begin{array}{c}
\mathbf{R} \\
\mathbf{0}_{2 \times 2}
\end{array}\right]
$$

where $\mathbf{Q}=\left[\begin{array}{ll}\mathbf{Q}_{1} & \mathbf{Q}_{2}\end{array}\right]$ is a unitary $4 \times 4$ matrix consisting of two $4 \times 2$ sub-matrices $\mathbf{Q}_{1}$ and $\mathbf{Q}_{2}, \mathbf{R}$ is a $2 \times 2$ upper triangular matrix with real-valued positive diagonal entries, and $\mathbf{0}_{2 \times 2}$ is a $2 \times 2$ zero matrix. Then,

$$
\begin{gathered}
\left\|\tilde{\mathbf{Y}}_{b}-\varphi_{c, b} \tilde{\mathbf{H}} \tilde{\mathbf{S}}_{b}\right\|^{2}=\left\|\tilde{\mathbf{Y}}_{b}-\varphi_{c, b}\left[\begin{array}{ll}
\mathbf{Q}_{1} & \mathbf{Q}_{2}
\end{array}\right]\left[\begin{array}{c}
\mathbf{R} \\
\mathbf{0}
\end{array}\right] \tilde{\mathbf{S}}_{b}\right\|^{2}= \\
\left\|\left[\begin{array}{c}
\mathbf{Q}_{1}^{*} \\
\mathbf{Q}_{2}^{*}
\end{array}\right] \tilde{\mathbf{Y}}_{b}-\varphi_{c, b}\left[\begin{array}{c}
\mathbf{R} \\
\mathbf{0}
\end{array}\right] \tilde{\mathbf{S}}_{b}\right\|^{2} \\
=\left\|\mathbf{Q}_{1}^{*} \tilde{\mathbf{Y}}_{b}-\varphi_{c, b} \mathbf{R} \tilde{\mathbf{S}}_{b}\right\|^{2}+\left\|\mathbf{Q}_{2}^{*} \tilde{\mathbf{Y}}_{b}\right\|^{2}
\end{gathered}
$$

We note that the second term in (11) is not a function of the symbols that need to be decoded. Due to the orthogonality of the Alamouti code and using the Gram-Schmidt method to calculate $\mathbf{Q}_{1}$ and $\mathbf{R}$, the conventionally modulated symbols for the specific SMP can then be decoded as

$$
\hat{\tilde{\mathbf{S}}}_{b}=\arg \min _{\mathbf{s}_{i, b} \in S} \sum_{i=1}^{2}\left\|\tilde{\mathbf{h}}_{i}^{H} \tilde{\mathbf{Y}}_{b}-\varphi_{c, b} \mathcal{E}_{H} \mathbf{s}_{i, b}\right\|^{2}
$$

where

$$
\mathcal{E}_{H}=\sum_{k=1}^{2} \sum_{l=1}^{2}\left|H_{k, l}\right|^{2}
$$

is the energy of the MIMO channel. Since the corresponding symbols in each of the sums in (12) are independent

$$
\hat{s}_{i, b}=\operatorname{demod}\left\{\frac{\tilde{\mathbf{h}}_{i}^{H} \tilde{\mathbf{Y}}_{b}}{\varphi_{c, b} \mathcal{E}_{H}}\right\} ; i=1,2 ; b=1, . ., B .
$$

In the equation above, $\operatorname{demod}\{R\}$ represents the typical constellation demodulator (i.e, slicer) which exploits the geometrical properties of the constellation to find the symbol closest to the point $R$ and thus avoids performing exhaustive search over all possible symbols.
Consequently, after estimating the corresponding symbols using (14) the $\mathcal{M}_{\min }(c)$ can be calculated using (9), for each SMP. Finally, the ML solution will appear as

$$
\hat{c}=\arg \min _{c \in \mathcal{C}} \mathcal{M}_{\min }(c) .
$$

The calculation of (14) for each $b=1, \ldots, B=L / 2$ requires 12 complex multiplications/divisions. In addition, the norm calculation in (9) requires 14 complex multiplications (if $\varphi_{c, b}$ is first multiplied with $\hat{\tilde{\mathbf{S}}}_{b}$ ). Therefore, the complexity is $13 L 2^{n_{s m}}$ complex multiplications, where $n_{s m}$ is the number of SMBs. However, independent of the number of the available SMPs and for each $b$ value, $\mathcal{M}_{\text {min }}$ can take only as many values as the number of the phase modulation states $\left(M_{S M}\right)$. Therefore, the complexity can be calculated to be $13 L M_{S M}$ complex multiplications. Therefore, the complexity of the proposed scheme, can be calculated as

$$
J_{p}=\min \left\{13 L 2^{n_{s m}}, 13 L M_{S M}\right\} .
$$

However, as it is later discussed, the $M_{S M}$ value can be kept low (e.g., $M_{S M}=2$ ), so the complexity can be kept manageable. For the simulation evaluations of Section IV, $M_{S M}=2$ is assumed.

For the decoding of the rateless coded data information, soft-information-based sum-product rateless decoder is employed. From Eq. 12 the Log-Likelihood Ratios (LLRs) of the CMBs can be cacluated as

$$
L_{\left(s_{i, b}\right)}=\ln \left(\frac{\sum_{s_{i, b} \in S_{b}^{0}} \exp \left[\frac{-\left\|\tilde{\mathbf{h}}_{i}^{H} \tilde{\mathbf{Y}}_{b}-\hat{\varphi}_{c, b} \mathcal{E}_{H} s_{i, b}\right\|^{2}}{2 \mathcal{E}_{H} \sigma_{n}^{2}}\right]}{\sum_{s_{i, b} \in S_{b}^{+1}} \exp \left[\frac{-\left\|\tilde{\mathbf{h}}_{i}^{H} \tilde{\mathbf{Y}}_{b}-\hat{\varphi}_{c, b} \mathcal{E}_{H} s_{i, b}\right\|^{2}}{2 \mathcal{E}_{H} \sigma_{n}^{2}}\right]}\right)
$$

where $\hat{\varphi}_{c, b}$ is the phase rotation obtained from SMP $\hat{c}$ (given by (15)), and $S_{b}^{0}$ and $S_{b}^{+1}$ are the subsets of possible symbols that have the $b^{t h}$ bit equal to 0 and +1 respectively.

\section{STSM FOR JOINT MEDIUM ACCESS AND RATELESS DATA TRANSMISSION}

This section describes how STSM can be used in the context of MTC for joint medium access and rateless data transmission. The scenario considered here assumes multiple machines that want to communicate with a central access point. However, the approach can be extended to machines that communicate with each other in a non-centralized way (e.g., ad-hoc machine-type networks). It is also assumed that the ACK messages transmitted from the access point take place using a dedicated channel in a broadcast manner.

As discussed, it is assumed that the transmission is ratelessly encoded. While the proposed approach is independent of the employed rateless scheme, Raptor codes [15] are employed here. The coded information is modulated, space-time-encoded and transmitted in sets of blocks of a size of $L$ symbols, as shown in Fig. 2. Small blocks are required so that the rateless systems can be close to the channel capacity. Each machine can transmit the blocks either in a continuous manner, or in a 

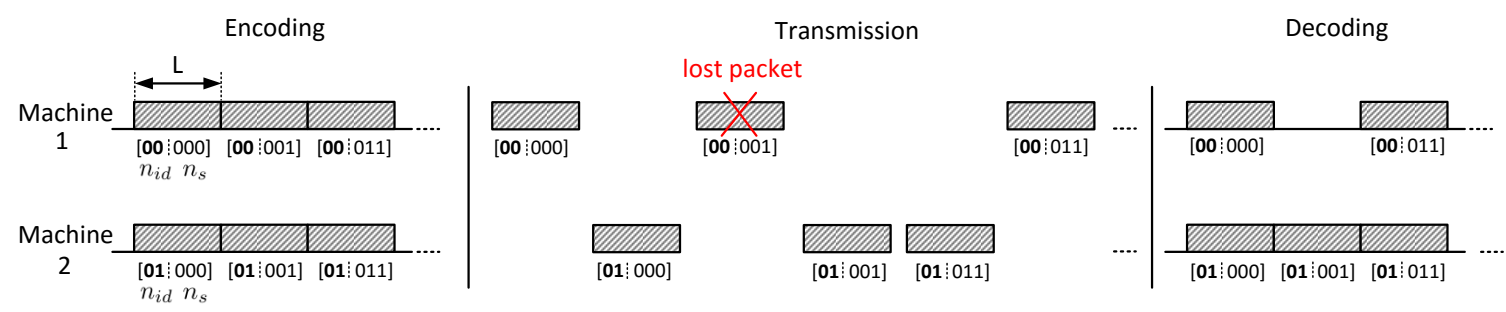

Fig. 2. Transmission of ratelessly coded information packets in a collision-free, multiuser environment.

random way, since the proposed approach supports both kinds of transmission. Each machine continues transmitting block related to the same information sequence, until it receives an ACK from the access point that the corresponding information sequence has been decoded. In order for the access point to decode the received information, it needs to know the ID of the machine that transmitted the packet, as well as its relative position in the encoded sequence (see Fig. 2). Therefore, together with each packet, some signature information needs to be transmitted. In the examined case, this signature information consists of two parts. The first set of $n_{i d}$ bits provides the ID of the transmitting machine and the second set of bits $n_{s}$ is used to provide the order of the transmitted packet in the encoding sequence. The $n_{i d}$ bits can be either preallocated to machines or they can be randomly selected as in the case of mobile RACH. The way to allocate them and the corresponding consequences, are beyond the scope of this work, which targets the reliable identification of the signature information in a collision-free environment.

Several approaches can be used to transmit all or part of the signature bits. The first approach transmits preambles packet before each data packet, with the preamble bits being encoded with some low rate code. Since advanced channel codes like LDPC codes are not appropriate for such small packet lengths, here traditional convolutional coding is assumed. In addition, it is assumed that the coded packets are also space-timeencoded. A second approach, used also in mobile RACH, transmits dedicated preamble sequences that are orthogonal to each other. Similarly to mobile (LTE) RACH, here preambles based on Zadoff-Chu sequences are considered [16] and the corresponding sizes are such that can support the number of bits to be mapped. Specifically, to transmit $q$ bits we need to map them to $2^{q}$ sequences of a length of at least $2^{q}$ transmission samples. Both these approaches require transmitting preambles, that as shown in Section IV can significantly limit the achievable rate. Instead, the SMBs of STSM can be used to transmit part of or the whole signature information, reducing or even eliminating the need for preambles.

\section{Evaluation}

In this section, the performance of STSM is evaluated via simulations. A $2 \times 2$ MIMO Rayleigh channel is assumed and the conventional information is BPSK modulated. In all evaluations, the transmitted power is normalized to unity. In all cases perfect channel knowledge at the receiver is assumed.

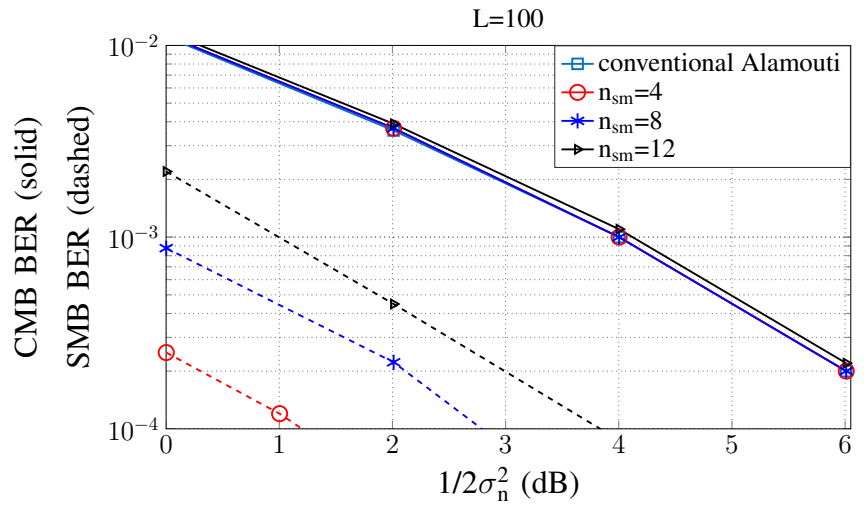

Fig. 3. BER performance of the CMBs and SMBs versus traditional Alamouti for several $n_{s m}$ values and $L=100$

Fig. 3, shows the uncoded bit-error-rate (BER) performance for the CMBs and SMBs, for $L=100$ and several SMB block sizes $n_{s m}$. It is shown that the error-rate performance of CMBs is better for smaller $n_{s m}$ values, while the performance of SMBs remains practically unaffected.

Fig. 4 compares the signature packet-error-rate performance when transmitting $n_{s i g}=4$ signature bits per packet, using several approaches. In particular, it compares the error-rate performance with STSM and packets of $L=200$ against an approach that utilizes Zadoff-Chu preambles of $N_{p r}=16$ samples (which is the minimum preamble that supports 4bit packets). For this case, it is assumed that the sequence is transmitted from one antenna, and at the receiver side coherent detection of the transmitted sequence takes place. In addition, the signature packet-error-rate performance of coded preambles of size $N_{p r}=16$ is shown, for different modulation sizes and code rates, and when they are Alamouti space-time encoded. It is shown that STSM outperforms all other cases despite the absence of a preamble.

Fig. 5 evaluates the achievable rate of one user in a collisionfree environment when using Raptor rateless codes for different methods to encode each packet's signature information (SI). Raptor's inner LT code is generated according to Raptor RFC 5053 standard [17], and rate 0.95 LDPC pre-code with left regular distribution (node degree 3 for all nodes) and right Poisson (check nodes chosen randomly with a uniform distribution) is also used. Belief propagation decoding is performed with forty iterations [15]. The SI of $n_{\text {sig }}=9$ bits 


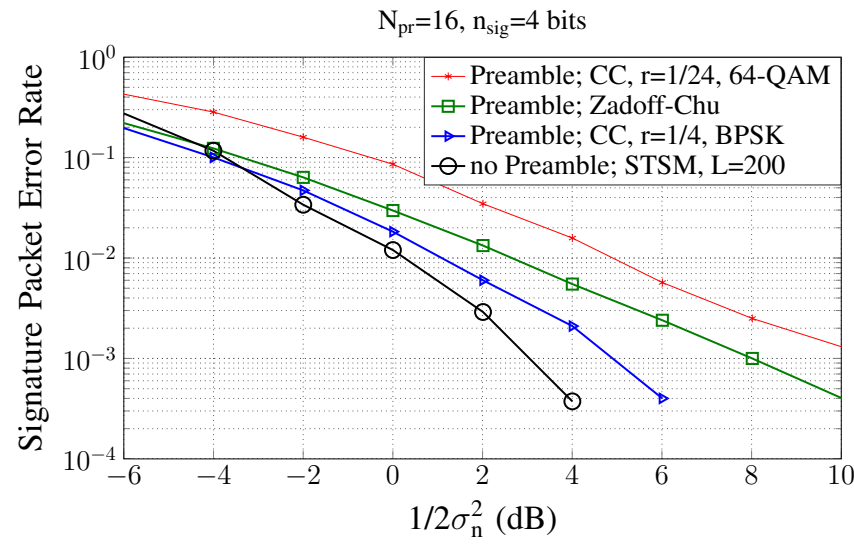

Fig. 4. Comparison of signature information packet error rate with $n_{\text {sig }}=4$ for STSM without preamble and $L=200$ against Zadoff-Chu preambles and convolutional coded (CC) preambles of size $N_{p r}=16$.

consists of $6 n_{i d}$ bits (to support 64 users similarly to the mobile RACH), and $3 n_{s}$ bits. Five cases are considered, all targeting the efficient delivery of the nine signature bits. The case where the signature information is perfectly known, the case where all signature bits are super-modulated (SM) and no preamble is used, the case where preambles of size $N_{p r}=64$ are used, that are BSPK modulated and convolutionally plus space-time encoded, the case where all bits are mapped onto a Zadoff-Chu Preamble (ZCP) (of $N_{p r}=512$ samples), the case of using two separate ZCPs for the $n_{i d}$ and the $n_{s}$ bits, of sizes $N_{p r, 1}=64$ and $N_{p r, 2}=8$ respectively, as well as the case where a ZCP $N_{p r}=64$ is used for mapping the $n_{i d}$ bits and the $n_{s}$ bits are super-modulated. In all cases, it is assumed that if the packets to be transmitted are more than those that can be counted by the $n_{s}$ bits, the counter is reset. In addition, if a signature packet sequence is found more than once, the most reliable (in terms of their soft metrics) is used. Fig. 5 shows that only STSM can approach the "ideal" rateless throughput in medium and large SNRs providing gains up to $45 \%$ compared to the all other solutions. In addition, in all SNRs STSM based solutions outperform solutions based solely on preambles. Still, it is noted that the reliability of the STSM approach can be further increased by increasing $L$.

\section{CONCLUSIONS}

In machine type communications with joint medium access and rateless data transmission, the use of preambles to transmit packet's signature information can significantly reduce the achievable rate. Here, a Space-Time Super-Modulation scheme is proposed that, by exploiting the structure of space-time block codes, can reduce or even eliminate the need for preambles resulting in throughput gains that for packets of 200 bits can be more than $45 \%$.

\section{ACKNOWLEDGMENT}

The authors would like to thank the members of University of Surrey 5GIC (http://www.surrey.ac.uk/5GIC) for their support.

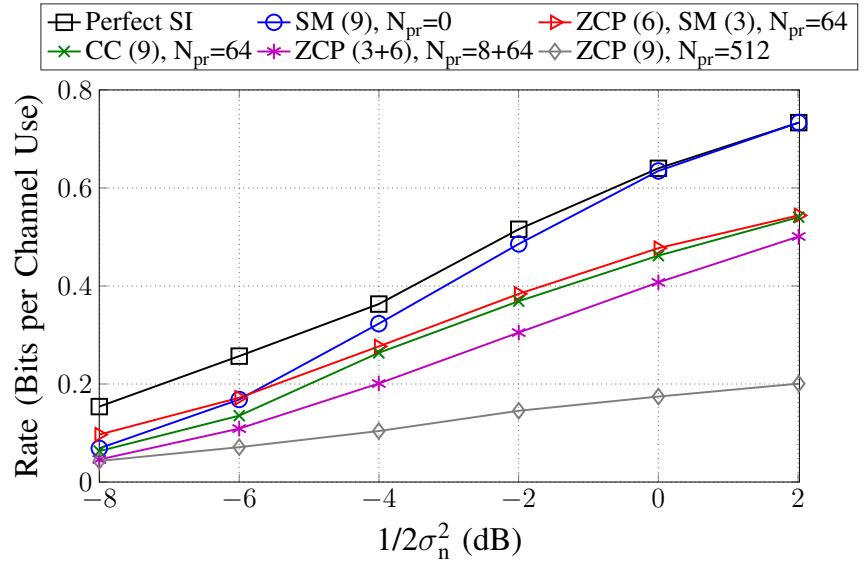

Fig. 5. Achievable rate of the rateless STSM scheme with $L=200$ versus preamble-based approaches.

\section{REFERENCES}

[1] H. Shariatmadari, et al., "Machine-type communications: current status and future perspectives toward 5G systems," IEEE Commun. Mag., vol. 53, no. 9, pp. 10-17, Sep. 2015.

[2] A. Rajandekar and B. Sikdar, "A survey of MAC layer issues and protocol for machine-to-machine communications," IEEE Internet Things J., vol. 2, no. 2, pp. 175-186, Apr. 2015.

[3] F. Ghavimi and H.-H. Chen, "M2M communications in 3GPP LTE/LTEA networks: architectures, service requirements, challenges, and applications," IEEE Commun. Surveys Tuts., vol. 17, no. 2, pp. 525-549, Second Quarter 2015.

[4] S.-Y. Lien, et al., "Toward ubiquitous massive accesses in 3GPP machineto-machine communications," IEEE Commun. Mag., vol. 49, no. 4, pp. 66-74, Apr. 2011.

[5] S. Andreev, et al., "Understanding the IoT connectivity landscape: a contemporary M2M radio technology roadmap," IEEE Commun. Mag., vol. 53, no. 9, pp. 32-40, Sep. 2015.

[6] G. Wunder, et al., "Sparse signal processing concepts for efficient 5G system design," IEEE Access, vol. 3, no. 2, pp. 195-208, Mar. 2015.

[7] "Final 5GNOW transceiver and frame structure concept D.3.3," 5GNOW Technical Report, May 2015.

[8] G. Wang, et al., "TiM: fine-grained rate adaptation in WLANs," IEEE Trans. Mobile Comput., vol. 3, no. 3, pp. 748-761, Mar. 2016.

[9] S. M. Alamouti, "A simple transmit diversity technique for wireless communications," IEEE J. Sel. Areas Commun., vol. 16, no. 8, pp. 14511458, Oct. 1998.

[10] V. Tarokh, H. Jafarkhani, and A. R. Calderbank, "Space-time block coding for wireless communications: performance results," IEEE J. Sel. Areas Commun., vol. 17, no. 3, pp. 451-460, Mar. 1999.

[11] _,'Space-time block codes from orthogonal designs," IEEE Trans. Inf. Theory, vol. 45, no. 5, pp. 1456-1467, Jul. 1999.

[12] U. Wachsmann, et al., "Multilevel codes: theoretical concepts and practical design rules," IEEE Trans. Inf. Theory, vol. 45, no. 5, 13611391, Jul. 1999

[13] G. Ungerboeck, "Trellis coded modulation with redundant signal sets, part I," IEEE Commun. Mag., vol. 25, pp. 5-11, Feb. 1987.

[14] H. Imai and S. Hirakawa, "A new multilevel coding method using error correcting codes," IEEE Trans. Inform. Theory, vol. 23, no. 3, pp. 371377, May 1977.

[15] O. Etesami and A. Shokrollahi, "Raptor codes on binary memoryless symmetric channels," IEEE Trans. Inf. Theory, vol. 52, no. 5, pp. 2033 2051, May 2006.

[16] H. S. Jang, et al., "Spatial group based random access for M2M communications," IEEE Commun. Lett., vol. 18, no. 6, pp. 961-964, Jun. 2014.

[17] M. Luby, A. Shokrollahi, M. Watson, and T. Stockhammer, "Raptor forward error correction scheme for object delivery," RFC 5053, Oct. 2007. 